\title{
Extensive demyelinating change in cerebrum after a total knee replacement -A case report-
}

\author{
Sang-Jin Park ${ }^{1}$, Seung-Dong Kim ${ }^{1}$, Dae-Lim Jee ${ }^{1}$, and Woo-Mok Byun ${ }^{2}$ \\ Departments of ${ }^{1}$ Anesthesiology and Pain Medicine, ${ }^{2}$ Diagnostic Radiology, College of Medicine, Yeungnam University, Daegu, Korea
}

Demyelination is characterized by the loss of myelin with the preservation of axons. Demyelinating diseases can be classified into several categories: demyelination due to inflammation, viral infection, osmotic derangements and hypoxic ischemia. In particular, osmotic myelinolysis is representative, and is associated with hyperosmolality, hypokalemia or rapid correction of hyponatremia. Osmotic myelinolysis was reported to be associated with underlying conditions, such as alcoholism, diuretics and malnutrition. A 67-year-old woman with hypertension was scheduled to undergo both total knee replacements (TKR). She was observed to be lethargic with dysphagia and quadriplegia after the second TKR. She had been taking diuretics for a long time, and did not have an adequate amount of food intake due to patient controlled analgesia and a gastric ulcer after the first TKR. A laboratory examination revealed hypokalemia but normonatremia. T2 weighted-MRI revealed abnormal high signal intensity in the basal ganglia and periventricular area. This case was diagnosed with osmotic myelinolysis associated with hypokalemia without an apparent sodium imbalance. (Korean J Anesthesiol 2010; 59: S197-S200)

Key Words: Demyelinating diseases, Hypokalemia, Osmotic myelinolysis, Total knee replacement.

Demyelinating diseases are disorders of the central nervous system (CNS) that involve an injury to myelins, not axons. They show a range of neurological symptoms depending on the lesion of invasion, such as speech disorders, convulsions, lethargy, quadriparesis, etc. The osmotic myelination associated with alcohol abuse, liver disease or a rapid correction of hyponatraemia is represented of demylinating diseases [1]. Many other diseases fall into this category: multiple sclerosis, acute disseminated encephalomyelitis, progressive multifocal leucoencephalopathy and hypoxic ischemic encephalopathy [1]. Therefore, a precise diagnosis of demyelinating diseases including the histopathology and radiology should be made clinically.

We encountered a patient with no specific disease except for hypertension who had an extensive demyelinating attack on the cerebrum, which appeared to be caused by osmotic demyelination after a total knee replacement (TKR). The diagnosis was quite difficult because few or no cases of CNS compli-

Received: June 11, 2010. Revised: 1st, July 8, 2010; 2nd, July 17, 2010. Accepted: August 8, 2010.

Corresponding author: Sang-Jin Park, M.D., Department of Anesthesiology and Pain Medicine, College of Medicine, Yeungnam University, Daemyeong-dong, Nam-gu, Daegu 705-030, Korea. Tel: 82-53-620-3369, Fax: 82-53-626-5275, E-mail: apsj0718@naver.com

(c) This is an open-access article distributed under the terms of the Creative Commons Attribution Non-Commercial License (http:// creativecommons.org/licenses/by-nc/3.0/), which permits unrestricted non-commercial use, distribution, and reproduction in any medium, provided the original work is properly cited. 
cations associated with TKR except for a cerebral infarction have been reported [2]. This paper reports the diagnosis, prevention and management of the demyelinating disease.

\section{Case Report}

A 67-year-old female patient was admitted to a local orthopedic hospital for TKR in both knees. She has been taking hydrochlorothiazide and lercanidipine for hypertension, and had been treated for a gastric ulcer 6 months earlier. The electrocardiogram, chest X-ray, blood analysis with electrolyte (WBC 8,900/ $\mu \mathrm{l}$, hemoglobin $13.1 \mathrm{~g} / \mathrm{dl}$, platelet $288,000 / \mu \mathrm{l}$, total protein $6.9 \mathrm{~g} / \mathrm{dl}$, albumin $4.2 \mathrm{~g} / \mathrm{dl}, \mathrm{Na} 142 \mathrm{mEq} / \mathrm{L}, \mathrm{K} 4.6 \mathrm{mEq} / \mathrm{L}$, $\mathrm{Cl} 106 \mathrm{mEq} / \mathrm{L}$ ) were within the normal limits. She was asked to take her antihypertensive drugs continuously.

First, a left TKR was performed. Spinal anesthesia was performed for surgery. Oxygen $2 \mathrm{~L} / \mathrm{min}$ was applied through a venturi mask and midazolam $2 \mathrm{mg}$ was injected. TKR was completed without problems. The operation and anesthetic time was 1 hour and 55 minutes and 2 hours and 20 minutes, respectively. Her vital signs were stable. Packed RBC (2 pints) and crystalloid $(700 \mathrm{ml})$ was administered intraoperatively. Patient-controlled analgesics (PCA), a mixture of butorphanol tartrate $(8 \mathrm{mg})$, ketorolac tromethamine $(180 \mathrm{mg})$ and ondansetron ( $8 \mathrm{mg}$ ), was connected to the intravenous line ( $1 \mathrm{ml} / \mathrm{hr}$ ). Additionally, the pain was controlled with intravenous diclofenac $\beta$-dimethyl aminoethanol and the oral administration of meloxicam $7.5 \mathrm{mg}$. The postoperative laboratory data revealed a WBC, hemoglobin level and platelet count of 8,900/ $\mathrm{ll}, 13.3 \mathrm{~g} / \mathrm{dl}$ and $194,000 / \mu \mathrm{l}$, respectively.

The patient complained of dizziness, nausea, vomiting and difficulty in eating a meal on the 3rd day after surgery. These symptoms improved after stopping PCA. The PCA was removed at the postoperative 5th day because the symptoms became aggravated after the PCA was restarted. However, gastrointestinal problems, such as heartburn, dyspepsia and newly-developed diarrhea, continued until the $10^{\text {th }}$ day after surgery. The patient was treated for gastric ulcer recurrence, and a liquid diet, such as gruel, was offered to her in this period. From 11th day after surgery, her symptoms improved and she was able to have a regular diet.

The electrolyte (Na $140 \mathrm{mEq} / \mathrm{L}, \mathrm{K} 3.6 \mathrm{mEq} / \mathrm{L}, \mathrm{Cl} 100 \mathrm{mEq} /$ L) and AST/ALT, BUN/Cr levels were within the normal limits at the day before the second operation. However, the patient had a fever of $38.6^{\circ} \mathrm{C}$ as well as chills, myalgia and coughing after taking a bath. Diclofenac $\beta$-dimethyl aminoethanol was administered intramuscularly, which relieved the symptoms. She also wanted to undergo the right TKR as planned. In the operating room, her blood pressure and heart rate was 160/90 $\mathrm{mmHg}$ and 90 beats/min, respectively. Spinal anesthesia was done to the anesthetic level of T10. Oxygen and midazolam were administered. She complained of a chilling sensation and her blood pressure increased to $185 / 90 \mathrm{mmHg}$. Pethidine HCL $25 \mathrm{mg}$ and hydralazine HCL $10 \mathrm{mg}$ were administered and the operation was completed without further problems. The operation time was 1 hour 50 minutes and 2 pints of packed RBC were administered. She had a heart rate, blood pressure and oxygen saturation of $80-95$ beats/ $\mathrm{min}, 185-150 / 90-70$ $\mathrm{mmHg}$ and $99 \%$, respectively, at the operating room and was transferred to the general ward. However, she showed nausea, vomiting and a fever of $39.5^{\circ} \mathrm{C}$. The symptoms were treated with antipyretics, metoclopramide HCL and tepid water massage. After that time, her body temperature went down to normal and the other symptoms disappeared.

On the morning of the day after the second operation, the caretaker of the patient complained of her being inarticulate. Her pronunciation was inaccurate and her verbal response was slow at the physical examination. She also underwent sedation gradually. At that time, her vital signs showed a blood pressure, heart rate and body temperature of 100/60 mmHg, 72 beats/ min and $36.6^{\circ} \mathrm{C}$, respectively. Multiple cerebral infarctions and extrapontine myelinolysis syndrome was indicated by brain magnetic resonance imaging (MRI). So the patient was transferred to other general hospital. In the emergency room, her consciousness faded out and self respiration was weakening and the endotracheal tube was intubated. Her caretaker requested transfer to our hospital again. When the patient arrived, she was intubated and in a stupor. All motor activities of the extremities evaluated were grade $1 / 5$. The laboratory findings showed the following: $\mathrm{Hb} 12.3 \mathrm{~g} / \mathrm{dl}$, serum $\mathrm{Na} 140 \mathrm{mEq} / \mathrm{L}, \mathrm{K} 2.9 \mathrm{mEq} / \mathrm{L}, \mathrm{Cl} 106 \mathrm{mEq} / \mathrm{L}$, total protein 5 $\mathrm{g} / \mathrm{dl}$ (normal range from 6.5 to $8.2 \mathrm{~g} / \mathrm{dl}$ ), albumin $2.8 \mathrm{~g} / \mathrm{dl}$

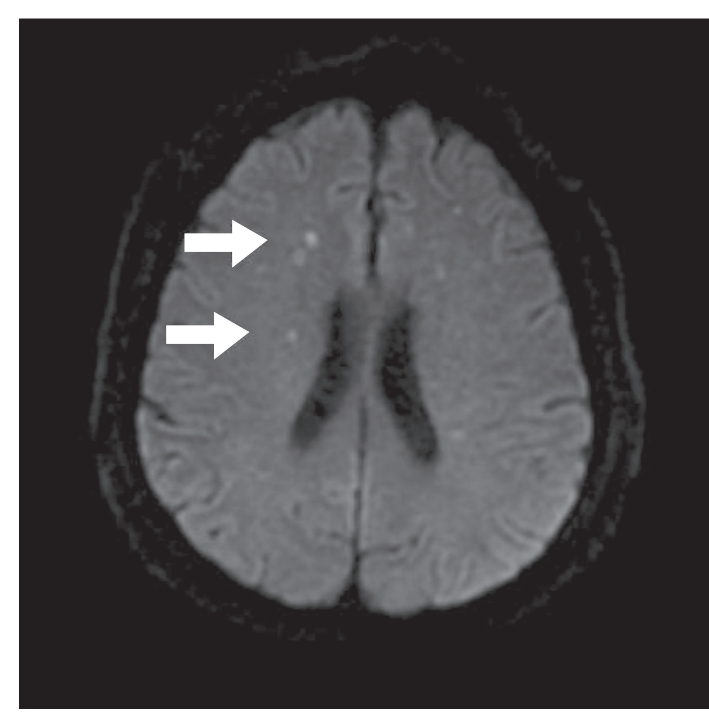

Fig. 1. Brain MRI shows a multiple cerebral infarction. 

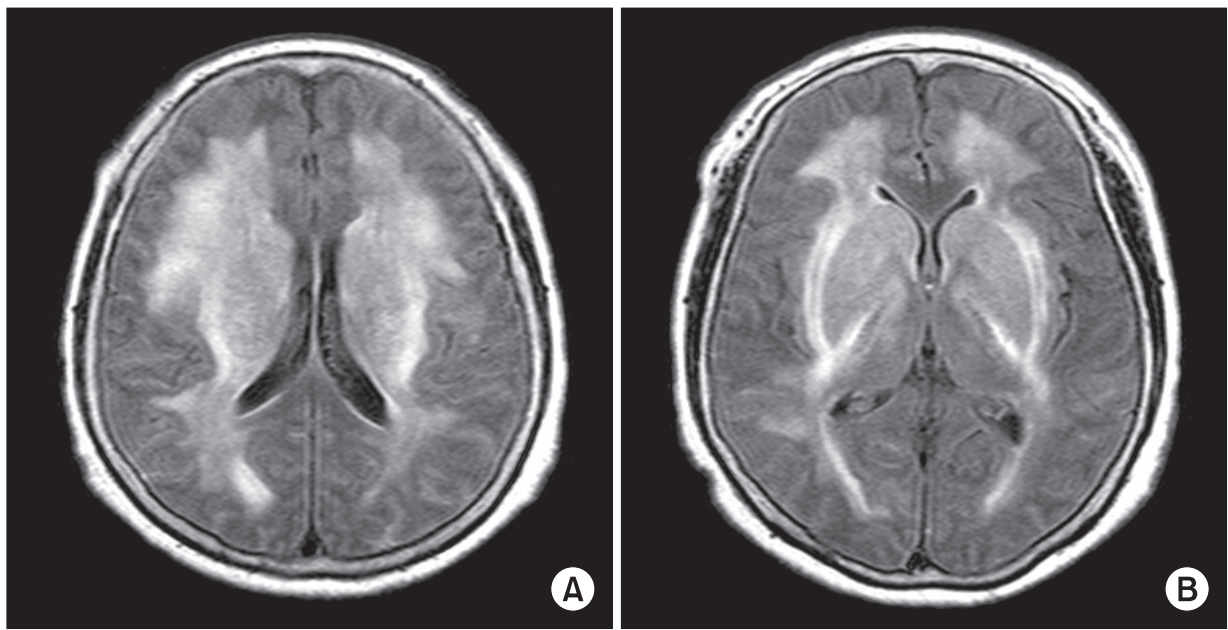

Fig. 2. (A) T2-weighted MRI of periventricular lesions with osmotic myelinolysis. High signal intensities are shown in the bilateral periventricular lesion. (B) Osmotic myelinolysis with bilateral basal ganglia involvement. T2-weighted MRI shows a symmetric high signal in both basal ganglia, with additional involvement of the subcortical white matter.

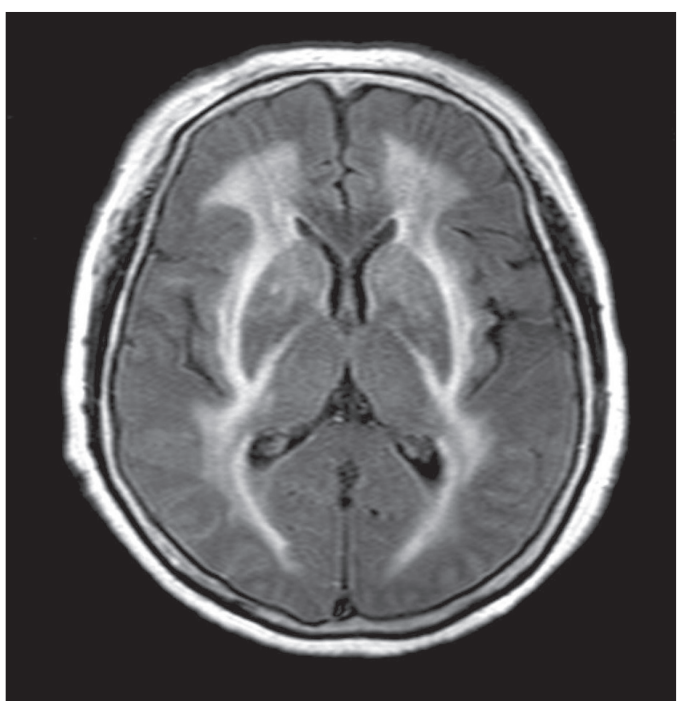

Fig. 3. T2-weighted MR image taken after 21 days shows a reduction of the hyperintense signal in the basal ganglia.

(normal range from 3.5 to $5 \mathrm{~g} / \mathrm{dl}$ ), and BUN/Cr 17.9/0.7 mg/ dl. A retaken MRI revealed acute multiple cerebral infarction (Fig. 1) and high signal intensity on both the periventricular area and basal ganglia in the T2-weighted image (Fig. 2). The radiologist suspected extrapontine myelinolysis syndrome. Methylprednisolone sodium succinate (methysol ${ }^{\circledR}$ ) was administered from two days in our hospital. The endotracheal tube was extubated on the 5th day. On the 18th day, she was able to talk and the motor activities of both upper extremities and lower extremities were $3 / 5$ and $1 / 5$ grade, respectively. On the 21th day, the right upper motor activity showed $4 / 5$ grade and MRI revealed a slight decrease in high intensity in both basal ganglia (Fig. 3). The patient kept improving gradually and was transferred to the department of rehabilitation for rehabilitation on the $48^{\text {th }}$ day.

\section{Discussion}

In the present case, the patient suffered lethargy, dysarthria and quadriparesis after TKR. MRI of the brain showed an acute multiple microinfarction, and increased signal intensity on both the periventricular area and basal ganglia in the T2-weighted images. These clinical images suggested that the patient's symptoms were consistent with osmotic demyelination invading the cerebrum extensively.

Osmotic demyelination is associated with hyperosmolality or a rapid correction of haponatremia. It has also been described in association with underlying conditions, such as alcoholism, malnutrition or liver transplantation, and exhibits symmetric hyperintensity on the T2-weighted image of brain MRI [3-5]. In this case, malnutrition might have developed because of the poor food intake due to PCA and gastric ulcer. In this situation, continuing diuretics use may also lead to an electrolyte imbalance. Indeed, the total protein and albumin were decreased to $5 \mathrm{~g} / \mathrm{dl}$ and $2.8 \mathrm{~g} / \mathrm{dl}$, respectively, on the day the neurologic symptoms occurred. These laboratory findings were consistent with malnutrition. The day before the second TKR, her potassium level was only $3.6 \mathrm{mEq} / \mathrm{L}$ despite having a regular diet and decreased to $2.9 \mathrm{mEq} / \mathrm{L}$ on the next day. Therefore, it is possible that hypokalemia developed after the first TKR. It had been reported that osmotic demyelination can develop without a significant change in sodium concentration in the presence of hypokalemia $[6,7]$. The taking of diuretics for long-term periods is also related to the development of osmotic myelination [3]. Moreover, her radiological findings, such as symmetric high signal intensity in the basal ganglia and periventricular area, were consistent with osmotic demyelination. The neurological symptoms were attributed to osmotic demyelination.

On the other hand, it is possible that there were other causes considering the patient had fever and coughing as well as 
myalgia in the two days before the neurological symptoms developed. Acute disseminated encephalomyelitis (ADEM) raises local neurological symptoms and encephalopathy within 2 days to 4 weeks after an infection or vaccination $[8,9]$. It can involve the basal ganglia and white matter of the cerebrum, brain stem, cerebellum and shows high signal intensity on the T2-weighted images, as in the present case $[8,9]$. However, ADEM is common in children and shows asymmetric, multiple and tumefactive lesions on the T2-weighted images $[9,10]$. Consequently, ADEM is less likely to be the cause of this case, even though the possibility of ADEM cannot be excluded completely.

Multiple sclerosis, another demyleinating disease, is affected strongly by heredity, and shows asymmetric and sporadical lesions with a clear border $[11,12]$. Progressive multifocal leucoencephalopathy is common in patients with impaired immune systems, such as AIDS or organ transplantation [13]. Hypoxic ischemic encephalopathy develops as a result of hypoxia due to cardiac arrest or carbon monoxide intoxication [14]. In this case, the radiological findings showed a symmetric lesion with an unclear margin. There were no immunosuppressed conditions or any events that could have caused the hypoxia. Hence, they are less likely to be the cause in this case.

The laboratory datas, radiologic findings, clinical symptoms and the biopsy of the lesion are essential for a precise diagnosis of demyelinating disease [1]. However, the condition was diagnosed without a biopsy because of the possibility of iatrogenic cerebral hemorrhage or neural injury. There were no problems with the diagnosis of osmotic demyelination, considering the underlying conditions, such as malnutrition and long term diuretics medication, an electrolyte imbalance and specific radiologic findings. It was also reported the osmotic demyelination was diagnosed without a direct biopsy of the lesion [6].

Steroid therapy is commonly used in osmotic demyelination as well as in other demyelinating diseases, such as acute disseminated encephalomyelitis and multiple sclerosis $[8,15]$. Therefore, steroid administration might have been appropriate for our patient. Nevertheless, the effect of steroid therapy has not been verified and the outcome varies from complete recovery to death [3]. Therefore, prevention may be more effective. In this case, the osmotic demyelination appeared to be associated with an electrolyte imbalance resulting from continuing diuretics use and malnutrition by PCA and gastric ulcer. The osmotic demyelination could have been prevented if the patient had been treated after identifying the electrolyte imbalance via blood analysis beforehand. In addition, the malnutrition would not have occurred if the NSAID had been restricted considering her gastric ulcer history.

In summary, this is a rare case of osmotic demyelination that developed after TKR, which involved hypokalemia without hyponatremia or associated rapid correction. The condition appeared to be related to malnutrition and the continuous use of diuretics. Clinicians need to be cognizant of the possibility of osmotic demyelination, even if the serum sodium concentration has not changed.

\section{References}

1. Love S. Demyelinating diseases. J Clin Pathol 2006; 59: 1151-9.

2. Parvizi J, Mui A, Purtill JJ, Sharkey PF, Hozack WJ, Rothman RH. Total joint arthroplasty: when do fatal or near-fatal complications occur? J Bone Joint Surg Am 2007; 89: 27-32.

3. Martin RJ. Central pontine and extrapontine myelinolysis: the osmotic demyelination syndromes. J Neurol Neurosurg Psychiatry 2004; 75 Suppl 3: iii22-8.

4. Roh JK, Nam H, Lee MC. A case of central pontine and extrapontine myelinolysis with early hypermetabolism on 18FDG-PET scan. J Korean Med Sci 1998; 13: 99-102.

5. Kang HS, Oh IY, Kim YJ, Cho CK, Lee SK, Yang SY, et al. Developed extrapotine myelinolysis after hysteroscopic myomectomy: a case report. Korean J Anesthesiol 2006; 50: 94-8.

6. Shintani M, Yamashita M, Nakano A, Aotani D, Maeda K, Yamamoto T, et al. Central pontine and extrapontine myelinolysis associated with type 2 diabetic patient with hypokalemia. Diabetes Res Clin Pract 2005; 68: 75-80.

7. Sugimoto T, Murata T, Omori M, Wada Y. Central pontine myelinolysis associated with hypokalaemia in anorexia nervosa. J Neurol Neurosurg Psychiatry 2003; 74: 353-5.

8. de Seze J, Debouverie M, Zephir H, Lebrun C, Blanc F, Bourg V, et al. Acute fulminant demyelinating disease: a descriptive study of 60 patients. Arch Neurol 2007; 64: 1426-32.

9. Tenembaum S, Chitnis T, Ness J, Hahn JS; International Pediatric MS Study Group. Acute disseminated encephalomyelitis. Neurology 2007; 68: S23-36.

10. Sonneville R, Klein I, de Broucker T, Wolff M. Post-infectious encephalitis in adults: diagnosis and management. J Infect 2009; 58: 321-8.

11. Sadovnick AD, Ebers GC, Dyment DA, Risch NJ. Evidence for genetic basis of multiple sclerosis. The Canadian Collaborative Study Group. Lancet 1996; 347: 1728-30.

12. Singh S, Prabhakar S, Korah IP, Warade SS, Alexander M. Acute disseminated encephalomyelitis and multiple sclerosis: magnetic resonance imaging differentiation. Australas Radiol 2000; 44: 404-11.

13. Sweet TM, Del Valle L, Khalili K. Molecular biology and immunoregulation of human neurotropic JC virus in CNS. J Cell Physiol 2002; 191: 249-56.

14. Lo CP, Chen SY, Lee KW, Chen WL, Chen CY, Hsueh CJ, et al. Brain injury after acute carbon monoxide poisoning: early and late complications. AJR Am J Roentgenol 2007; 189: W205-11.

15. Hagiwara K, Okada Y, Shida N, Yamashita Y. Extensive central and extrapontine myelinolysis in a case of chronic alcoholism without hyponatremia: a case report with analysis of serial MR findings. Intern Med 2008; 47: 431-5. 